\title{
Inhalt
}

GÜNTHER SCHULZ

Zur Einführung

HANS HECKER

Vertreibung und Verfolgung in der jüngeren deutschen Geschichte

WOLFGANG KESSLER

Elitenwechsel. Die Gebietsabtretungen in Posen-Westpreußen und

Oberschlesien 1920-1922 und die regionalen Führungsschichten

CLAUS-DIETER KROHN

Vertriebene intellektuelle Eliten aus dem

nationalsozialistischen Deutschland

HILTRUD HĀNTZSCHEL

Die Konkurrenz um „die wahre deutsche Kultur“. Vertriebene

kulturelle Eliten aus dem nationalsozialistischen Deutschland.

MARITA KRAUSS

Die Rückkehr einer vertriebenen Elite. Remigranten

in Deutschland nach 1945

ARND BAUERKÄMPER

Vertreibung als Exklusion gesellschaftlicher Führungsgruppen.

Die Verdrängung der „Großbauern“ in der SBZ/DDR und

die Vernichtung der „Kulaken“ in der UdSSR im Vergleich.

MiCHAEL SCHWARTZ

„Verantwortliche Arbeit beim Wiederaufbau“. Die Vertriebenen

und die Formation neuer administrativer Eliten in der SBZ/DDR

MATHIAS BEER

Die Vertreibung der Deutschen aus Ost-Mitteleuropa und die politischadministrative Elite der Bundesrepublik. Ein Problemaufriss 


\section{REINHARD BUTHMANN}

Abwanderung und Flucht von Eliten aus der SBZ/DDR

am Beispiel der wissenschaftlichen Intelligenz.

HANS-WERNER RAUTENBERG

Die Vertreibung aus dem deutschen Osten und die kulturelle Elite. 267

Die Autorinnen und Autoren des Bandes 